\title{
Politique
}

\section{Pour un mode de scrutin équitable. La proportionnelle territoriale. Rapport de la commission de la représentation électorale, 28 mars 1984, 199 p.}

\section{Robert Boily}

Numéro 6, automne 1984

La réforme des institutions politiques

URI : https://id.erudit.org/iderudit/040465ar

DOI : https://doi.org/10.7202/040465ar

Aller au sommaire du numéro

Éditeur(s)

Société québécoise de science politique

ISSN

0711-608X (imprimé)

1918-6584 (numérique)

Découvrir la revue

Citer ce compte rendu

Boily, R. (1984). Compte rendu de [Pour un mode de scrutin équitable. La proportionnelle territoriale. Rapport de la commission de la représentation électorale, 28 mars 1984, 199 p.] Politique, (6), 167-173.

https://doi.org/10.7202/040465ar

Ce document est protégé par la loi sur le droit d'auteur. L'utilisation des services d'Érudit (y compris la reproduction) est assujettie à sa politique d'utilisation que vous pouvez consulter en ligne.

https://apropos.erudit.org/fr/usagers/politique-dutilisation/ 


\section{RECENSIONS}

Pour un mode de scrutin équitable. La proportionnelle territoriale. Rapport de la commission de la représentation électorale, 28 mars 1984, 199 p.

Au début de l'été 1983, une Assemblée nationale peu tentée par une réforme du mode de scrutin donnait, à l'unanimité, mandat à la Commission de la représentation électorale d'évaluer les avantages et les inconvénients du mode de scrutin actuel, d'étudier les diverses formules proposées, tenir des audiences publiques à cette fin et si elle le jugeait nécessaire, la Commission pouvait soumettre des recommandations. Par la suite, les députés boudèrent les audiences de la Commission et accueillirent avec mauvaise humeur le rapport lorsqu'il fut déposé, dans les délais prévus, le 28 mars 1984.

Rien de nouveau ne pouvait être vraiment dit sur les inconvénients et les avantages du mode de scrutin actuel. La question avait été retournée en tous sens plus d'une fois depuis plus de quinze ans à l'intérieur comme à l'extérieur du Parlement. Au moment où la Commission a été saisie de son mandat, ce n'était plus un problème d'information qu'affrontaient le gouvernement et l'Assemblée nationale mais un problème de décision. Aussi la Commission a-t-elle eu raison de ne pas consacrer beaucoup d'espace de son rapport à ce qui était déjà connu et facilement accessible. Elle a plutôt préféré donner en toute franchise son avis sur la nécessité d'un changement par l'introduction d'un élément proportionnel dans notre manière de convertir les suffrages en sièges. Cette Commission a également, dans le contexte d'une réforme 
à froid, fait tout son possible, compte tenu de ses moyens, pour informer et intéresser la population à cette question. Elle aura permis ainsi à de nombreux groupes de s'exprimer. On peut regretter cependant que l'excellente synthèse du rapport, présentée en une dizaine de pages au début de celui-ci n'ait pas connu une diffusion plus large.

Il ne faut donc pas chercher dans ce rapport une information détaillée sur les principaux modes de scrutin comme dans le livre vert du ministre Robert Burns. La Commission se contente également de résumer les principaux arguments en faveur ou contre le système actuel et de présenter, de manière succincte mais didactique, les caractéristiques des divers systèmes proposés lors des audiences. L'essentiel du rapport, le plus intéressant, c'est l'exposé des paramètres dont il faut tenir compte dans l'analyse d'un mode de scrutin au Québec, l'explicitation de son choix et les quelques propositions que la Commission avance touchant les principaux problèmes de la mise en place d'une propositionnelle au Québec.

La Commission a identifié cinq principaux thèmes auxquels il faut s'attacher lors de l'étude d'un mode de scrutin soit, la représentation des électeurs, la délimitation territoriale, le rôle du député et la vie parlementaire, la stabilité gouvernementale et le rôle des partis politiques. Pour chacun de ces points, le rapport fournit un état de la question et un bilan des interventions. C'est à travers ces divers états de la question que la Commission laisse percer sa manière de voir, les fondements de son choix. Si son point de vue demeure assez conservateur, traditionnel et parfois étroit sur le rôle du député dans ses relations avec les électeurs et dans son activité parlementaire - ainsi, croire que c'est l'évolution du parlementarisme qui a amené les députés à accentuer leur rôle de représentant, d'intermédiaire, c'est sous-estimer totalement l'influence beaucoup plus déterminante de la nature de nos partis politiques - la Commission aborde avec beaucoup plus de largeur 
de vue, de nuances, la question de la stabilité gouvernementale, apportant sur ce point des distinctions importantes entre stabilité gouvernementale et stabilité des institutions. On n'y retrouve pas cette obsession de la stabilité gouvernementale à tout prix, de cette stabilité ramenée à l'existence d'un gouvernement majoritaire et entraînant à coup sûr efficacité et progrès. C'est cette même largeur de vue que l'on retrace dans sa volonté de respecter le pluralisme existant qu'il soit ethnique, linguistique ou idéologique.

Les réponses que la Commission a apportées à ces diverses questions l'ont amenée à opter pour une forme de proportionnelle, mais une proportionnelle qu'elle entend ancrer dans la réalité québécoise. Or, cette réalité est beaucoup plus complexe qu'on ne le croit souvent: pluralisme des options politiques et sociales, présence de minorités ethniques et culturelles et notamment des autochtones mais aussi une distribution très particulière de la population suite au type d'urbanisation et d'industrialisation qu'a vécu le Québec. En effet, le Québec connaît à la fois de fortes concentrations de population, pensons à la région de Montréal et de Québec, et une dispersion marquée de sa population dans des régions périphériques. Tenant compte de ces réalités, la Commission déclare que s'il faut assurer la majorité de la représentation au monde urbain, il faut également compenser les problèmes de distance et de communication de ces régions périphériques par une représentation plus élevée que ce qu'autoriserait une stricte proportionalité.

L'agencement de tous ces traits ne donne pas pour autant naissance à des réalités régionales au sens sociologique, comme le suppose le projet du ministre Bédard ou encore celui de Vincent Lemieux. C'est donc à raison selon nous que les commissaires ont refusé d'asseoir leur réforme sur une telle croyance et préféré parler de territoires.

La Commission a également voulu s'éloigner des projets de proportionnelle régionale modérée en cherchant à mieux composer 
majorité gouvernementale et reflet des tendances au sein de la société québécoise, à mieux faciliter l'évolution politique par une plus juste représentation des courants nouveaux. Ce n'est que partiellement cependant que l'objectif est atteint. Alors que le projet Bédard comme celui de Vincent Lemieux prévoient des circonscriptions variant de trois à sept députés, ce qui constitue un obstacle de taille à l'expression de tendances autres que majoritaires, la Commission propose pour les régions fortement urbanisées des circonscriptions élisant plus de dix députés. Les membres de la Commission illustrent leur recommandation à partir de deux hypothèses de découpage du territoire.

Une première hypothèse prévoit un total de 125 sièges avec des circonscriptions variant de 3 à 14 députés. Une deuxième retient une possibilité de 127 sièges à partir de circonscriptions permettant l'élection de 3 à 19 députés. L'effet indéniable de ce type de propositions est donc d'augmenter par rapport à la proposition du ministre Bédard les possibilités de tendances nouvelles extérieures aux partis dominants. Il faut cependant réaliser que dans l'une ou l'autre hypothèse, cette possibilité n'est ouverte que dans les milieux fortement urbains de Montréal et de Québec (hypothèse I, 36 sièges; hypothèse II, 43 sièges), soit le quart de la représentation. Notons de plus que dans la deuxième hypothèse, la création de telles circonscriptions plus larges s'accompagne d'un accroissement du nombre de circonscriptions dans lesquelles ne seraient élus que de 3 à 4 députés (lère hypothèse, 6 circonscriptions; 2ème hypothèse, 13 circonscriptions).

Il est difficile de croire que le dynamisme et le pluralisme du Québec se réfugient dans les deux seuls grands centres urbains. Comment pourront faire entendre leurs voix dans ces circonscriptions de la périphérie qui ne permettront d'élire que trois députés, ces groupes souvent minoritaires aux prises avec des problèmes pourtant majeurs. Le Québec réel vit une complexité qui déborde ces frontières artificielles où l'on veut l'enfermer. Le pluralisme ethnique 
et linguistique de Montréal n'est qu'une facette de ce monde complexe que nous habitons. On ne peut ainsi, à l'avance, décider des lieux où doit s'exprimer le dynamisme social et politique.

La Commission va un peu plus avant et propose un certain nombre de modalités qui pourraient accompagner la mise en place d'un nouveau mode de scrutin. De fait deux de ces propositions ont pour effet de réduire l'objectif d'une plus juste représentation des tendances et réalités du Québec. En effet, en préférant situer au niveau de chacun des territoires découpés l'opération de transformation des voix en sièges et cela en utilisant la formule d'Hondt, soit celle du plus fort reste, la Commission retient deux modalités réductrices déjà présentes dans les projets Bédard et Lemieux. De tels choix accentuent le caractère modéré de la réforme proposée. Il y a ici rétrécissement au niveau des modalités des effets attendus à partir de ces objectifs annoncés.

Fidèle à sa lecture des principaux paramètres impliqués par une réforme du mode de scrutin, la Commission estime que si les partis doivent établir les listes présentées aux électeurs, il revient à ces derniers d'en établir l'ordre définitif. L'électeur doit donc pouvoir opter pour un parti et pour des candidats. Si nous partageons l'idée de mettre fin aux élections partielles en prévoyant le remplacement d'un député démissionnaire ou mort par un substitut choisi au moment de l'élection - les sondages renseignent amplement les gouvernements sur l'état de l'opinion, rôle que jouaient entre autres auparavant les élections partielles - nous ne pouvons accepter qu'une telle mesure entraîne pour un député l'impossibilité de quirter son parti et de siéger comme indépendant. On augmente là indûment le pouvoir des partis, à qui on accorde déjà celui de dresser l'ordre des listes et de bénéficier de la discipline de vote à l'Assemblée nationale. On entre de plus en contradiction avec d'autres préférences exprimées comme celle de la possibilité de choix pour l'électeur de bouleverser l'ordre de la liste et le souci maintes fois rappelé de préserver la relation députés-électeurs. 
Ne vaudrait-il pas mieux explorer du côté d'une intervention des électeurs qui ne soit pas une élection partielle, plutôt que de brimer la possibilité pour un député de porter un jugement sur l'orientation du parti auquel, devant ses électeurs et à l'occasion de la demande de leur appui, il a donné son adhésion. Ce malaise à l'égard des indépendants se retrouve face à la manière de pénaliser le vote attribué à un tel type de candidature. Alors que l'on affirme donner à un indépendant le statut de parti, on ne reconnaît pas de fait à un électeur qui décide de voter pour un indépendant la même liberté de choix que lorsqu'il vote pour un parti. Il s'agit à nouveau d'une modalité réductrice de la reconnaissance de la pluralité des tendances.

Le lecteur trouvera enfin dans ce rapport une série d'annexes: cartes illustrant les hypothèses de découpage, mandats donnés à la Commission, mémoire des Cris de la Baie de James, etc. Il y retrouvera aussi un historique du mode de scrutin au Québec qui rappelle fort pertinemment au lecteur une série de faits et de données. On peut toutefois déplorer la manière toute aseptique de faire cet historique, une aseptie qui finit par entraîner une vue tronquée de l'évolution historique de notre système électoral. Il est difficile de laisser croire que de 1791 à aujourd'hui le nombre de députés s'est accru parallèlement à celui de la population sans que n'interviennent gerrymandering et manipulations de toutes sortes. Comment expliquer qu'en 1830 , pour une population de près de 550,000 personnes il y avait 84 députés et 85 en 1923 pour une population de plus de 2,360,000? Je ne crois pas que le caractère non-partisan de certaines institutions publiques ou parapubliques les oblige à déformer l'histoire. On aurait pu éviter également, dans un document dont la qualité d'information est plutôt la marque distinctive, d'écrire qu'en 1867 la constitution de la Province de Québec fut intégrée à l'A.A.B.N. alors que cette colonie n'existait plus depuis 1840 et que son intégration 
au Canada uni avait eu précisément comme objectif de lui dénier toute personnalité constitutionnelle.

En conclusion, l'observateur que je suis doit reconnaître que dans des circonstances difficiles, dans un délai fort court, la Commission est parvenue, dans un style clair, accessible à tous, à articuler une proposition de réforme appuyée sur une réflexion intéressante par plus d'un point. Ce rapport méritait entre autre de la part des députés plus de respect que ce qui fut exprimé. Ce fut une erreur de la part de la Commission de décider de ne pas intervenir une fois son rapport déposé. Si elle n'avait pas à entrer dans le débat partisan, elle se devait de répondre aux déformations de ses positions, de son analyse. L'homme engagé que je suis, qui depuis près de vingt ans appelle la réforme d'un mode de scrutin ne peut que regretter le caractère modéré de cette réforme, reflet, à côté de vues plus courageuses, de conceptions traditionnelles. Faute de mieux, la modétation a peut-être meilleur goût! L'analyste et l'homme engagé ne peuvent cependant que rappeler que le choix d'un mode de scrutin ne relève pas uniquement d'un choix entre les qualités dites objectives parce qu'abstraites d'un contexte donné mais d'un choix éminemment politique fondé sur la lecture d'une société, une lecture forcément idéologique.

Robert Boily

Université de Montréal 